\title{
Interlimb Coordination of Ground Reaction Forces during Double Stance Phase at Fast Walking Speed
}

\author{
Yoichiro Sato ${ }^{1,2 *}$, Norimasa Yamada ${ }^{3}$ \\ ${ }^{1}$ Department of Physical Therapy, Faculty of Health Sciences, Hokkaido University of Science, Sapporo, Japan \\ ${ }^{2}$ Laboratory of Human Movement Science, Graduate School of Education, Hokkaido University, Sapporo, Japan \\ ${ }^{3}$ Department of Sport and Physical Education, School of Health and Sport Sciences, Chukyo University, Toyota, Japan \\ Email: ${ }^{*}$ sato-y@hus.ac.jp
}

How to cite this paper: Sato, Y., \& Yamada, N. (2018). Interlimb Coordination of Ground Reaction Forces during Double Stance Phase at Fast Walking Speed. $A d$ vances in Physical Education, 8, 263-273. https://doi.org/10.4236/ape.2018.82024

Received: April 5, 2018

Accepted: May 26, 2018

Published: May 29, 2018

Copyright @ 2018 by authors and Scientific Research Publishing Inc. This work is licensed under the Creative Commons Attribution International License (CC BY 4.0).

http://creativecommons.org/licenses/by/4.0/

(c) (i) Open Access

\begin{abstract}
To better understand interlimb coordination during the double stance phase at fast walking speeds, we analyzed ground reaction forces generated by the leading and trailing limbs during the double stance phase at multiple speeds. Ground reaction forces were recorded during the double stance phase at slow, self-selected, and fast walking speeds in eleven healthy volunteers. We calculated the instantaneous phase of the ground reaction forces for the vertical and anterior-posterior components, and then calculated the relative phase between the leading and trailing limbs for each component. For the vertical component, the relative phase showed a significantly lower value in the fast condition than in the other two conditions in the early-double stance phase (fast vs. self-selected, $\mathrm{p}<0.01$; fast vs. slow, $\mathrm{p}<0.001$ ). For the anterior-posterior component, the relative phases in the early- and late-double stance phases in all speed conditions were significantly smaller than those in the mid-double stance phase. These findings suggest that interlimb coordination of the forces exerted by the leading and trailing limbs in the early-double stance phase would be an important factor for walking at fast speed.
\end{abstract}

\section{Keywords}

Fast Walking Speed, Interlimb Coordination, Double Stance Phase, Relative Phase, Healthy Young Adults

\section{Introduction}

During a double stance phase in human walking, the center of mass (COM) has a transition from the trailing limb to the leading limb, and the direction of the 
COM changes from downward to upward. The COM transitions consume most of energy expended during the entire gait cycle (Donelan et al., 2002; Kuo, 2007; Umberger, 2010). Furthermore, COM redirections require energy (Adamczyk \& Kuo, 2009). Thus, energy losses due to ineffective coordination between the leading and trailing limbs during the double stance phase should directly affect the energy efficiency of walking.

Interlimb coordination during the double stance phase has been observed spatially (Soo \& Donelan, 2010; Soo \& Donelan, 2012; Sousa et al., 2013) and temporally (Kuo, 2002; Sousa et al., 2012; Sato \& Yamada, 2017). From the spatial point of view, when muscle activity for absorbing an impact on the leading limb decreases, that for the forward propulsion of the trailing limb can increase (Sousa et al., 2013). Additionally, it was reported that a decrease in the push-off forces of the trailing limb increases the collision forces of the leading limb (Soo \& Donelan, 2012).

In the temporal aspects, Sato and Yamada (2017) calculated the relative phases of each component of the ground reaction forces (GRFs) between the leading and trailing limbs. This was achieved by calculating the instantaneous phase of each component in the leading and trailing limbs during the double stance phase at self-selected speed. They found that the relative phases of vertical component showed an anti-phase for smooth transitions from the trailing limb to the leading limb, and that anterior-posterior (AP) component showed a lag during entire double stance phase. These findings suggested that the forces generated by the leading and trailing limbs temporally coordinate to translate the COM as smoothly as possible.

Mechanical works performed on the COM during walking depend upon walking speed (Willems, Cavagna, \& Heglund, 1995). The COM works for walking are minimal at the self-selected speed, and increase as walking speed increases (Cavagna et al., 1976; Cavagna \& Kaneko, 1977). The muscle activities of lower limbs during walking also depend on walking speed (Hof et al., 2002). Muscle activities for body support and forward propulsion during the double stance phase increase as walking speed increases (Sousa \& Taveras, 2012). In clinical situations, it was considered that subjects with conditions such as hemiplegia cannot walk faster owing to the lack of these muscle activities (Olney \& Richards, 1996; Sousa \& Taveras, 2015). However, questions remain regarding why some post-stroke subjects cannot walk faster (Sousa et al., 2013).

Although we hypothesized that impaired interlimb coordination during the double stance phase might explain why some individuals cannot walk faster, to our knowledge, no study has examined how changes in walking speed affect coordination, even with healthy subjects as a reference. Hence, the purpose of the present study is to elucidate how different walking speeds affect the relative phases of the GRFs between the leading and trailing limbs in healthy subjects, in order to clarify interlimb coordination at fast walking speeds. The findings from this study will be useful for assessing gait ability in clinical situations. 


\section{Methods}

\subsection{Participants}

Eleven healthy males participated in this study. The mean ( \pm one standard deviation [SD]) age of the subjects was $24.3 \pm 3.7$ years; the average height and mass of the subjects were $1.76 \pm 0.06 \mathrm{~m}$ and $74.1 \pm 12.1 \mathrm{~kg}$, respectively. Inclusion criteria were that volunteers were healthy and had exhibited no clinical gait abnormalities during the past year, and that the dominant leg was on the right side. Before the experiments began, the volunteers read a description of the basic procedures of the experiments and provided informed consent. The experimental procedures of this study were approved by the ethics committee of Hokkaido University in Sapporo, and were carried out in accordance with the principles outlines in the Declaration of Helsinki for experiments involving humans.

\subsection{Procedures}

We instructed each subject to walk on a 5-m walkway at three different speeds: self-selected, fast, and slow. The subjects walked at their preferred speed at first, and then randomly walked at slow speed or fast speed. Under preferred speed condition, we instructed them to walk at their preferred speed. Under slow or fast conditions, we instructed them to walk slower or faster, respectively, than their preferred speed.

Each subject performed at least three trials under each condition. The GRFs of each subject were measured using two separate force platforms (OR6-5, AMTI, MA, USA; FP4060, Bertec Co. OH, USA) that were ground-embedded in a series near the midpoint of the 5-m walkway. All subjects could see the two platforms. The first force platform was touched by the left foot and the second one by the right foot. The measured GRFs were simultaneously digitized into two force dimension components (Fz: vertical; Fx: AP) using an analog-digital converter (16 bits; National Instrument, TX, USA) at $1000 \mathrm{~Hz}$. The positive values of the vertical component corresponded to the upward direction, and the positive values of the AP component corresponded to the forward direction. A trial was rejected if each foot did not clearly touch each force platform. We saved and analyzed the data for three acceptable trials for each subject. Data for each subject was obtained by averaging data from the three trials.

\subsection{Data Analysis}

The GRFs were smoothed using a zero-lag low-pass filter at $25 \mathrm{~Hz}$ (Bryant et al., 1984; Soo \& Donelan, 2010). We defined a double stance phase as the time between ground contact of the right foot (leading limb) and the foot-off of the left foot (trailing limb).

To calculate the instantaneous phase of the vertical component in each limb, we deconstructed the vertical component of each limb $\left(P G_{L V}\right.$ and $\left.P G_{T V}\right)$ into real and imaginary parts by using the Hilbert transform (Boashash, 1992a; Boashash, 1992b), formulated as Equation (1): 


$$
\begin{aligned}
& P G_{L V}(t)=G_{L V}(t)+i G H_{L V}(t) \\
& P G_{T V}(t)=G_{T V}(t)+i G H_{T V}(t)
\end{aligned}
$$

where subscripts $L$ and $T$ represent the leading and trailing limbs, respectively, and subscript $V$ represents the vertical component of the GRF. Moreover, $i$ represents the imaginary unit, and $G H_{L V}$ and $G H_{T V}$ represent the values of the vertical component in the leading and trailing limbs, respectively, transformed by the Hilbert transform. The instantaneous phase of the AP component of the GRF in each limb was calculated in a similar manner as the vertical component. The relative phases of the vertical component $\left(\Delta \Phi_{v}\right)$ and AP component $\left(\Delta \Phi_{A P}\right)$ were calculated using the real and imaginary parts of each component, formulated as Equations (2) and (3), respectively (Palut \& Zanone, 2005; Lames, 2006; Okumura et al., 2012):

$$
\begin{gathered}
\Delta \Phi_{v}=\tan ^{-1} \frac{G H_{L V} \cdot G_{T V}-G H_{T V} \cdot G_{L V}}{G_{L V} \cdot G_{T V}+G H_{L V} \cdot G H_{T V}} \\
\Delta \Phi_{A P}=\tan ^{-1} \frac{G H_{L A P} \cdot G_{T A P}-G H_{T A P} \cdot G_{L A P}}{G_{L A P} \cdot G_{T A P}+G H_{L A P} \cdot G H_{T A P}}
\end{gathered}
$$

where $G H_{L A P}$ and $G H_{T A P}$ represent the values of the AP component in the leading and trailing limbs, respectively, transformed by the Hilbert transform.

The double stance phase in each trial was divided into 10 sections (Sato \& Yamada, 2017). Walking speeds differed among the three conditions. Moreover, because the speeds also differed among subjects, we could not distinguish when the relative phases differed among the conditions during the double stance phase. The mean of values in each section was used as the representative value for that section. All computations were performed in MATLAB (MathWorks Inc., MA, USA).

\subsection{Statistical Analysis}

The durations of the double stance phase under self-selected, fast, and slow conditions were examined using the repeated-measures one-way analysis of variance (ANOVA). The effect size of ANOVA, $\eta^{2}$, was calculated. Repeated-measures two-way ANOVAs were performed as factors of the walking speed and the sections to examine the relative phases of the vertical and AP components of the GRF. A Tukey's honest significance difference (HSD) was performed as a post-hoc test. All statistical analyses were performed using SPSS version 20.0 (IBM Corporation, Armonk, NY, USA). The statistical significance level was set as 0.05 .

\section{Results}

The double stance phase durations were, on an average (SDs) of all subjects, as follows: 113.7 (18.6) $\mathrm{ms}, 158.1$ (21.9) $\mathrm{ms}$, and 195.4 (20.8) $\mathrm{ms}$ under fast, self-selected, and slow conditions, respectively (Figure 1). The main effect of the walking speeds was significant $(\mathrm{F}(2.30)=43.65, p<0.001)$, and there were 


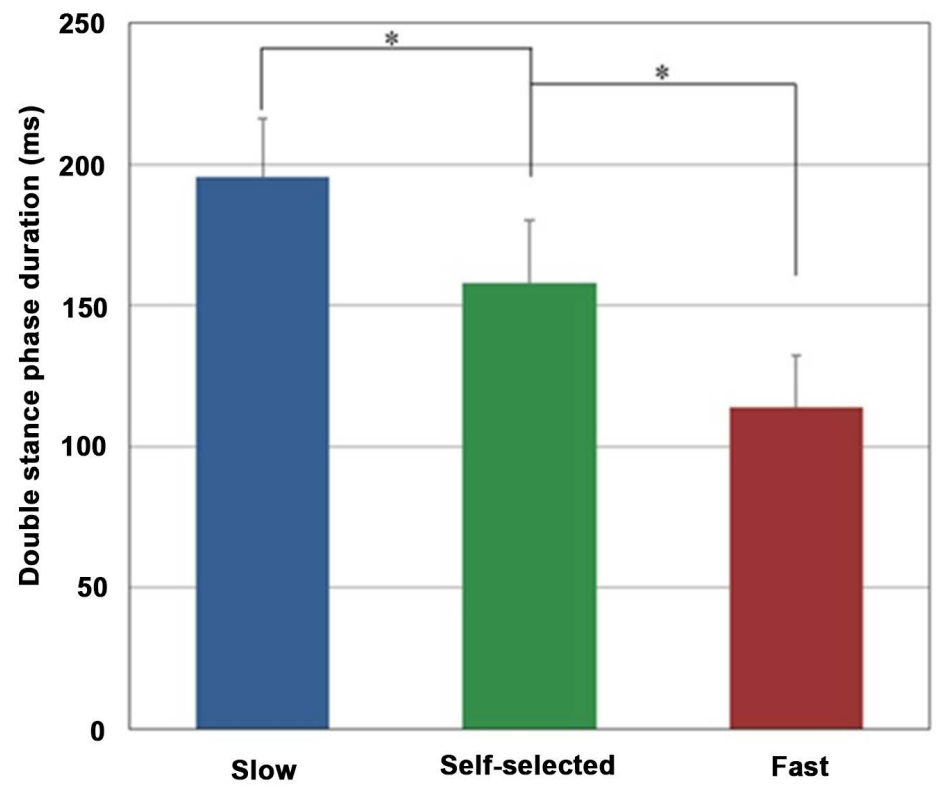

Figure 1. Mean of the duration of the double stance phase under each condition for all subjects $(\mathrm{n}=11)$. The error bars show one standard deviation. ${ }^{*}: p<0.001$.

significantly differences between the walking speed conditions $(p<0.001)$. The effect size of ANOVA was 0.744 .

The vertical component of the GRF during the double stance phase showed a crossed pattern for the leading and trailing limbs under all walking speeds (Figure 2(a)).

The increase in the force generated by the leading limb showed more nonlinearity in the fast condition than in the other conditions (around $30 \mathrm{~ms}$ in Figure 2(a)). For the AP component, the positive (i.e., push-off) force generated by the trailing limb decreased; the force generated by the leading limb showed a positive force at the early-double stance phase and then a negative (i.e., impact) force (Figure 2(b)).

Table 1 shows the relative phases of the vertical component of the GRF in each section. The interaction of the walking speed and the section was significant $(\mathrm{F}(18,300)=2.95, p<0.001)$. The main effect of the walking speed was significant $(\mathrm{F}(2,300)=11.49, p<0.001)$. Its relative phase under the fast condition was significantly different from the other two conditions (fast vs. self-selected, $p$ $<0.01$; fast vs. slow, $p<0.001)$. The main effect of the section was also significant $(\mathrm{F}(9,300)=13.61, p<0.001)$. The Tukey's HSD test showed that its relative phases in DS2 and DS3 were significantly lower than in the other sections $(p<$ 0.001).

For the AP component of the GRF (Table 2), the main effect of only the section was significant $(\mathrm{F}(9,300)=3.17, p<0.01)$. The relative phase was lower in DS1 than in DS4 and DS5 $(p<0.05)$, and the relative phase in DS10 was lower than that in DS4 $(p<0.05)$. 


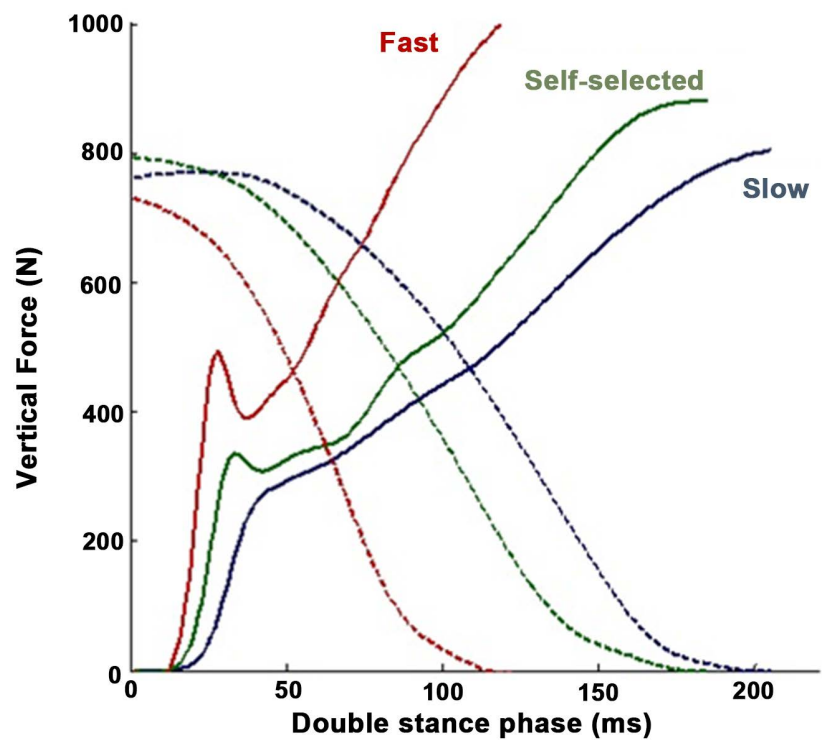

(a)

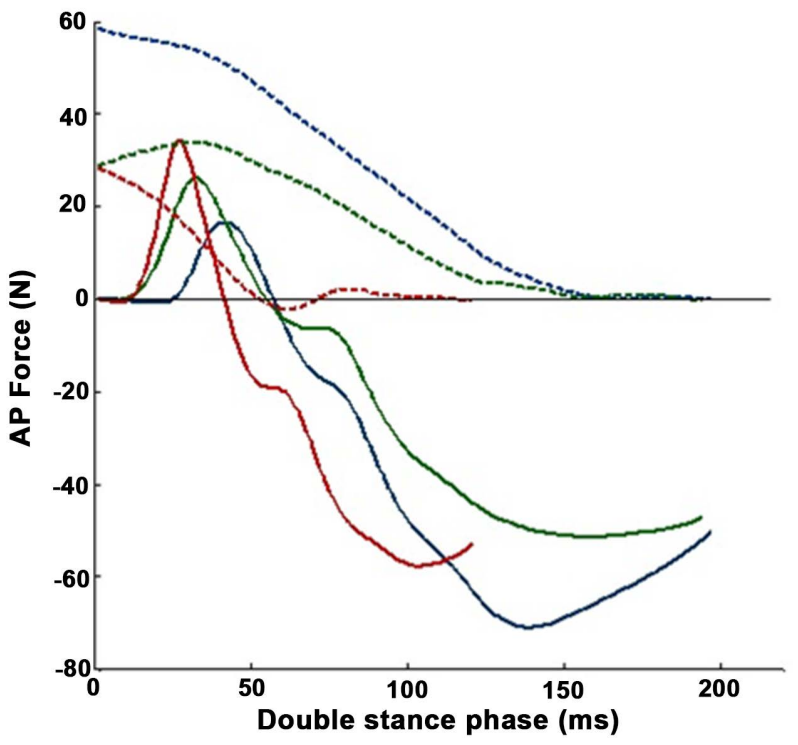

(b)

Figure 2. (a) Typical time course for the vertical component of GRF during the double stance phase under each condition; (b) Typical time course for the AP component of GRF during the double stance phase under each condition. Each line shows the value averaged across the three trials of each subject. Solid and dashed lines represent the leading and the trailing limbs, respectively, under each condition. Blue lines show the slow condition. Green lines represent the self-selected condition. Red lines represent the fast condition.

Table 1. Relative phase for the vertical component of each section in each condition $\left(\mathrm{n}=11^{\circ}\right)$.

\begin{tabular}{ccccccccccccc}
\hline & & DS1 & DS2 $\dagger$ & DS3 $\dagger$ & DS4 & DS5 & DS6 & DS7 & DS8 & DS9 & DS10 \\
\hline Slow & MEAN & 168.5 & 154.5 & 162.3 & 166.7 & 166.7 & 171.4 & 170.6 & 170.9 & 172.9 & 175.2 \\
& SE & 1.2 & 2.9 & 3.8 & 3.3 & 3.7 & 1.8 & 1.1 & 1.1 & 1.1 & 1.1 \\
Self & MEAN & 168.3 & 149.0 & 161.7 & 162.3 & 165.4 & 170.1 & 170.7 & 167.9 & 168.7 & 172.9 \\
& SE & 1.3 & 4.1 & 4.8 & 6.0 & 6.2 & 4.1 & 1.9 & 1.8 & 1.8 & 1.3 \\
\multirow{2}{*}{ Fast $^{*}$} & MEAN & 172.0 & 153.9 & 142.1 & 162.6 & 164.8 & 163.0 & 167.2 & 167.3 & 167.7 & 170.6 \\
& SE & 0.5 & 2.1 & 5.4 & 4.0 & 4.1 & 4.5 & 3.3 & 2.0 & 1.9 & 1.5 \\
\hline
\end{tabular}

*: significant difference compared to Slow and Self conditions $(p<0.001)$. $\dagger$ : significant difference compared to the other eight conditions (vs. Slow, $p<$ 0.001 ; vs. Self-selected, $p<0.01$ ).

Table 2. Relative phase for the AP component of each section in each condition $\left(\mathrm{n}=11^{\circ}\right)$.

\begin{tabular}{ccccccccccccc}
\hline & & DS1 & DS2 & DS3 & DS4 & DS5 & DS6 & DS7 & DS8 & DS9 & DS10 $\dagger$ \\
\hline \multirow{2}{*}{ Slow } & MEAN & 27.2 & 36.6 & 47.5 & 61.9 & 55.3 & 57.6 & 55.7 & 46.2 & 36.9 & 30.9 \\
& SE & 8.0 & 8.2 & 8.9 & 12.2 & 12.9 & 11.0 & 10.6 & 10.5 & 9.0 & 7.1 \\
\multirow{2}{*}{ Self } & MEAN & 33.2 & 43.3 & 46.0 & 49.9 & 53.9 & 48.4 & 49.4 & 46.6 & 42.2 & 35.3 \\
& SE & 6.8 & 7.8 & 13.5 & 13.2 & 12.2 & 10.9 & 10.9 & 11.1 & 10.0 & 8.0 \\
\multirow{2}{*}{ Fast } & MEAN & 33.0 & 47.5 & 52.0 & 46.5 & 44.7 & 40.9 & 42.6 & 44.5 & 37.6 & 27.3 \\
& SE & 4.8 & 9.4 & 12.1 & 8.0 & 8.5 & 8.2 & 8.7 & 7.7 & 6.9 & 4.9 \\
\hline
\end{tabular}

*: significant difference compared to DS4 and DS5 $(p<0.05)$. $\uparrow$ : significant difference compared to DS4 $(p<0.05)$. 


\section{Discussion}

The present study analyzed how varying walking speeds affect the relative phase between the leading and trailing limbs during the double stance phase. The vertical component of the GRF showed that the relative phase under the fast condition differs from that under the self-selected and slow conditions; in particular, the relative phase during the early to mid-double stance phase (DS3) under fast conditions was low (significant interaction). In contrast, the AP component showed no difference in the relative phase among the speed conditions.

The low relative phase during the early-double stance phase in the fast condition $\left(140^{\circ}-150^{\circ}\right)$ may indicate that an interlimb coordination during this phase is an important factor for walking faster. Sato and Yamada (2017) reported that the relative phase for the vertical component in subjects' preferred walking speed showed a value of approximately $180^{\circ}$ (i.e., anti-phase). They described that this finding indicates smooth transition of the COM from the trailing limb to the leading limb. Hence, no anti-phase of the relative phase in the fast condition in the present study suggests that the COM cannot translate smoothly as walking faster. Non-smooth translations occurring at normal walking speeds may be a factor because of which elderly or disabled individuals could not walk at fast walking speeds. Further investigation is necessary to clarify this hypothesis.

Furthermore, non-smooth translation of the COM in the fast condition may be associated with the ability to absorb the impact force of a leading limb on the floor during an early-double stance phase. In this phase, the leading limb absorbs the impact force (Perry, 1992; Rose \& Gamble, 2006). In smooth translations of the COM with an absorption of the impact force, the vertical component of the GRF, as shown in the slow (blue line) and self-selected conditions (green line) of Figure 2(a), can increase linearly. In contrast, as walking speed increases, because the impact forces increase as walking speeds increase (Kim \& Park, 2011; Yoem \& Park, 2011), nonlinearity in increases of the vertical component becomes considerable (Cavanagh \& Lafortune, 1980), as shown in the red line of Figure 2(a). This nonlinearity indicates that the COM bounds when the leading limb cannot absorb the impact force. Thus, the present finding suggests that, even among healthy young adults, the COM bounds with an increase in walking speed because the leading limb cannot absorb the impact force. A walking model simulation showed a nonlinear increase in the vertical component of the GRF as the stiffness of the leading leg was increased (Geyer et al., 2006). Elderly or disabled individuals show high leg stiffness during early-double stance phase (Hortobagyi \& DeVita, 2000). Thus, because they have limited ability to absorb impact forces during this phase, they may show a nonlinear GRF increase during this phase, even in normal walking velocity. Future research with elderly or disabled individuals would be necessary to verify this observation.

The present finding may be available for an assessment of gait ability in clinical situations. Factors responsible for the inability of patients, such as post-stroke, to perform fast speed walking are still not clear (Sousa et al., 2013). 
The present finding suggests that the relative phase between the leading and trailing limbs at an early-stance phase may explain these factors. The subjects in the present study were healthy adults. Although only the fast condition showed a significant difference in the relative phase in the present study, patients such as post-stroke may exhibit a low relative phase even under self-selected speed. Future studies would be needed to examine the relative phase of the vertical component in such patients.

The relative phase of the AP component of the GRF showed no significant differences between different walking speeds (Table 2). Because spatial parameters such as step length and muscle activities in lower limbs relating to forward propulsion are influenced by the walking speed (Sousa \& Tavares, 2012; Sekiya et al., 1997; Sekiya \& Nagasaki, 1998), we hypothesized that the relative phase under the self-selected condition would show characteristic values. However, the present study found that the relative phase of the AP component was not influenced by the walking speed. Relative phases cannot reflect the magnitude of the measured GRF, but can reflect the relationships of the increase and decrease of the force between the leading and trailing limbs. The present finding indicates that, even under the fast walking speed, relationships between them do not vary in normal subjects. Hence, because the collision and push-off forces increase with increases in walking speed (Kim \& Park, 2011; Yoem \& Park, 2011), this finding would indicate that individuals must control these varying forces without changing relative phases.

In the sections during the double stance phase, the relative phase was lower at DS1 than that at DS4 and DS5 (Table 2). As shown in Figure 2(b), forces generated by both the leading and trailing limbs showed positive values at DS1. The lower relative phase of two forces moving in a forward direction indicates that those forces contribute to forward propulsion together (Sato \& Yamada, 2017). Previous studies reported that the AP component of the GRF in the leading limb shows only negative impact force at ground contact (Donelan et al., 2002; Whittington \& Thelen, 2009). To the best of our knowledge, no previous study has described the positive forces observed in the present study. During the double stance phase, the fact that the trailing limb can generate positive push-off force is beyond doubt (Perry, 1992; Oastin, 2004). If the leading limb generates only negative forces (i.e., deceleration force) at the early-double stance phase, forces generated by the leading and trailing limbs will cancel each other. The cancelled forces do not show low relative phases. Thus, this finding showed that the leading limb can generate a positive force at the early-double stance phase. Future studies are necessary to clarify how this positive force is generated. Furthermore, abnormal coordination during the double stance phase in subjects with stroke was observed when the leading limb was the affected limb and the trailing limb was the unaffected limb (Sousa et al., 2013). The abnormal coordination suggests that the positive force generated by the leading limb could be an important factor for increasing walking speed in subjects with stroke. The relative phases of the AP component of the GRF in subjects with stroke should be investigated in 
future studies.

Regulated step lengths might be a limitation of the present study. Although walking speeds varied with changes in conditions in this study, step lengths were regulated by the dimensions of the force plates. Step lengths are also one of the factors affecting the magnitude of the GRF (Donelan et al., 2002; Kuo, 2007). Furthermore, because step length is directly proportional to walking speed (Sekiya et al., 1997; Kuo, 2001), regulating walking speed is similar to regulating step length. This limitation of the present study might have affected its results. However, as in previous studies, the dimensions of the force plates always affect subject performance (Donelan et al., 2002; Adamczyk \& Kuo, 2009). From this point of view, the findings of the present study would depend on walking speeds.

\section{Acknowledgements}

The authors thank Mr. Takuya IWATA and Mr. Shingo NATORI for helping us acquire the data. We also thank Editage (http://www.editage.jp) for scrutinizing the English.

\section{References}

Adamczyk, P. G., \& Kuo, A. D. (2009). Redirection of Center-of-Mass Velocity during the Step-to-Step Transition of Human Walking. Journal of Experimental Biology, 212, 2668-2678. https://doi.org/10.1242/jeb.027581

Boashash, B. (1992a). Estimating and Interpreting the Instantaneous Frequency of a Signal-Part 1: Fundamentals. Proceedings of the Institute of Electrical and Electronics Engineers, 80, 520-538. https://doi.org/10.1109/5.135376

Boashash, B. (1992b). Estimating and Interpreting the Instantaneous Frequency of a Signal-Part 2: Algorithms and Applications. Proceedings of the Institute of Electrical and Electronics Engineers, 80, 540-568. https://doi.org/10.1109/5.135378

Bryant, J. T., Wevers, H. W., \& Lowe, P. J. (1984). Method of Data Smoothing for Instantaneous Center of Rotation Measurements. Medical and Biological Engineering and Computing, 22, 597-602. https://doi.org/10.1007/BF02443876

Cavagna, G. A., \& Kaneko, M. (1977). Mechanical Work and Efficiency in Level Walking and Running. Journal of Physiology, 268, 467-481., https://doi.org/10.1113/jphysiol.1977.sp011866

Cavagna, G. A., Thys, H., \& Zamboni, A. (1976). The Sources of External Work in Level Walking and Running. Journal of Physiology, 262, 639-657. https://doi.org/10.1113/jphysiol.1976.sp011613

Cavanagh, P. R., \& Lafortune, M. A. (1980). Ground Reaction Forces in Distance Running. Journal of Biomechanics, 13, 397-406. https://doi.org/10.1016/0021-9290(80)90033-0

Donelan, J. M., Kram, R., \& Kuo, A. D. (2002). Simultaneous Positive and Negative External Mechanical Work in Human Walking. Journal of Biomechanics, 35, 117-124. https://doi.org/10.1016/S0021-9290(01)00169-5

Geyer, H., Seyfarth, A., \& Blickhan, R. (2006). Compliant Leg Behavior Explains Basic Dynamics of Walking and Running. Proceedings of the Royal Society B, 273, 2861-2867. https://doi.org/10.1098/rspb.2006.3637 
Hof, A. L., Elzinga, H., Grimmius, W., \& Halbertsma, J. P. K. (2002). Speed Dependence of Averaged EMG Profiles in Walking. Gait and Posture, 16, 78-86. https://doi.org/10.1016/S0966-6362(01)00206-5

Hortobagyi, T., \& DeVita, P. (2000). Muscle Pre- and Coactivity during Downward Stepping Are Associated with Leg Stiffness in Aging. Journal of Electromyography and Kinesiology, 10, 117-126. https://doi.org/10.1016/S1050-6411(99)00026-7

Kim, S., \& Park, S. (2011). Leg Stiffness Increases with Speed to Modulate Gait Frequency and Propulsion Energy. Journal of Biomechanics, 44, 1253-1258. https://doi.org/10.1016/j.jbiomech.2011.02.072

Kuo, A. D. (2001). A Simple Model of Bipedal Walking Predicts the Preferred Speed-Step Length Relationship. Journal of Biomechanical Engineering, 123, 265-269. https://doi.org/10.1115/1.1372322

Kuo, A. D. (2002). Energetics of Actively Powered Locomotion Using the Simplest Walking Model. Journal of Biomechanical Engineering, 124, 113-120. https://doi.org/10.1115/1.1427703

Kuo, A. D. (2007). The Six Determinants of Gait and the Inverted Pendulum Analogy: A Dynamic Walking Perspective. Human Movement Science, 26, 617-656. https://doi.org/10.1016/j.humov.2007.04.003

Lames, M. (2006). Modelling the Interaction in Game Sports-Relative Phase and Moving Correlations. Journal of Sports Science and Medicine, 5, 556-560.

Oastin, C. A. (2004). Kinesiology: The Mechanics and Pathomechanics of Human Movement. Philadelphia, PA: Lippincott Williams \& Wilkins.

Okumura, M., Kijima, A., Yokoyama, K., Suzuki, H., \& Yamamoto, Y. (2012). A Critical Interpersonal Distance Switches between Two Coordination Modes in Kendo Matches. PLoS ONE, 7, e51877. https://doi.org/10.1371/journal.pone.0051877

Olney, S. J., \& Richards, C. (1996). Hemiparetic Gait Following Stroke. Part 1: Characteristics. Gait and Posture, 4, 136-148. https://doi.org/10.1016/0966-6362(96)01063-6

Palut, Y., \& Zanone, P. G. (2005). A Dynamical Analysis of Tennis: Concepts and Data. Journal of Sports Science, 23, 1021-1032. https://doi.org/10.1080/02640410400021682

Perry, J. (1992). Gait analysis: Normal and Pathological Function. Thorofare: SLACK Inc.

Rose, J., \& Gamble, J. G. (2006). Human Walking (3rd ed.). Philadelphia, PA: Lippincott Williams \& Wilkins.

Sato, Y., \& Yamada, N. (2017). Temporal Coordination between Ground Reaction Forces Generated by Leading and Trailing Limbs for Propulsion during Double Stance Phase in Human Walking. Gait and Posture, 54, 295-299.

https://doi.org/10.1016/j.gaitpost.2017.03.026

Sekiya, N., \& Nagasaki, H. (1998). Reproducibility of the Walking Patterns of Normal Young Adults: Test-Retest Reliability of the Walk Ratio (Step-Length/Step-Rate). Gait and Posture, 7, 225-227. https://doi.org/10.1016/S0966-6362(98)00009-5

Sekiya, N., Nagasaki, H., Ito, H., \& Furuna, T. (1997). Optimal Walking in Terms of Variability in Step Length. Journal of Orthopedic and Sports Physical Therapy, 26, 266-272. https://doi.org/10.2519/jospt.1997.26.5.266

Soo, C. H., \& Donelan, J. M. (2010). Mechanics and Energetics of Step-to-Step Transitions Isolated from Human Walking. Journal of Experimental Biology, 213, 4265-4271. https://doi.org/10.1242/jeb.044214

Soo, C. H., \& Donelan, J. M. (2012). Coordination of Push-Off and Collision Determine the Mechanical Work of Step-to-Step Transitions When Isolated from Human Walking. Gait and Posture, 35, 292-297. https://doi.org/10.1016/j.gaitpost.2011.09.102 
Sousa, A. S. P., Silva, A., \& Tavares, J. M. R. (2012). Biomechanical and Neurophysiological Mechanisms Related to Postural Control and Efficiency of Movement: A Review. Somatosensory and Motor Research, 29, 131-143. https://doi.org/10.3109/08990220.2012.725680

Sousa, A. S. P., Silva, A., \& Tavares, J. M. R. (2013). Interlimb Relation during the Double Support Phase of Gait: An Electromyographic, Mechanical and Energy-Based Analysis. Proceedings of the Institution of Mechanical Engineers, Part H: Journal of Engineering in Medicine, 227, 327-333. https://doi.org/10.1177/0954411912473398

Sousa, A. S., \& Tavares, J. M. R. (2012). Effect of Gait Speed on Muscle Activity Patterns and Magnitude during Stance. Motor Control, 16, 480-492. https://doi.org/10.1123/mcj.16.4.480

Sousa, A. S., \& Tavares, J. M. R. (2015). Interlimb Coordination during Step-to-Step Transition and Gait Performance. Journal of Motor Behavior, 47, 563-574. https://doi.org/10.1080/00222895.2015.1023391

Sousa, A. S., Silva, A., Santos, R., Sousa, F., \& Tavares, J. M. R. (2013). Interlimb Coordination during the Stance Phase of Gait in Subjects with Stroke. Archives of Physical Medicine and Rehabilitation, 94, 2515-2522. https://doi.org/10.1016/j.apmr.2013.06.032

Umberger, B. R. (2010). Stance and Swing Phase Costs in Human Walking. Journal of the Royal Society Interface, 7, 1329-1340. https://doi.org/10.1098/rsif.2010.0084

Whittington, B. R., \& Thelen, D. G. (2009). A Simple Mass-Spring Model with Roller Feet Can Induce the Ground Reactions Observed in Human Walking. Journal of Biomechanical Engineering, 131, Article ID: 011013. https://doi.org/10.1115/1.3005147

Willems, P. A., Cavagna, G. A., \& Heglund, N. C. (1995). External, Internal and Total Work in Human Locomotion. Journal of Experimental Biology, 198, 379-393.

Yoem, J., \& Park, S. (2011). A Gravitational Impulse Model Predicts Collision Impulse and Mechanical Work during a Step-to-Step Transition. Journal of Biomechanics, 44, 59-67. https://doi.org/10.1016/j.jbiomech.2010.08.024 